Notes

\title{
Fluorometric Fiber-Optic Determination of Low Amounts of Water in Ethanol
}

\author{
Yoshiaki KuRAUCHI and Kazuya OHgA \\ Department of Environmental Chemistry and Engineering, Faculty of Engineering, Oita University, \\ Dannoharu, Oita 870-11, Japan
}

Keywords Water determination, fluorometric fiber-optic sensor, plasticized poly(vinyl chloride), acetal formation, 1-pyrenecarboxaldehyde

Water in organic solvents is generally determined by a gas-chromatographic technique ${ }^{1,2}$ or by Karl Fischer titration. Though these methods have low detection limits below $1 \mathrm{ppm}$, they are difficult to apply to onstream analysis. An optical sensor for water determination based on surface plasmon resonance ${ }^{3}$ has recently been developed in order to overcome this disadvantage. However, the concentration determined is relatively high (0.3-10\% in ethanol).

The acetal formation of 1-pyrenecarboxaldehyde (PCA) in ethanol followed by the hydrolysis of 1pyrenecarboxaldehyde diethyl acetal results in significant changes in the absorption and fluorescence spectra (described later). The spectral changes are expected to take place in a transparent plasticized poly(vinyl chloride)(PVC) membrane, which can take up water.,5 This paper describes the fabrication and characterization of a fluorometric fiber-optic sensor with a PVC membrane containing PCA as a probe to water in ethanol.

\section{Experimental}

\section{Reagents}

PCA was obtained from Aldrich Chem. Co. and recrystallized from benzene. High-molecular-weight PVC was purchased from Sigma Chem. Co. o-Nitrophenyl octyl ether (NPOE) and spectral grade tetrahydrofuran (THF) from DOJINDO Laboratories were used as received. Ethanol was distilled in the presence of $\mathrm{CaH}_{2}$ and dried over 3A molecular sieves (Wako Pure Chem. Industries). Acidic ethanol was prepared by bubbling gaseous $\mathrm{HCl}$ into ethanol. The acid concentration was determined from the difference in the density before and after bubbling. All other chemicals were of reagent grade or better and used without further purification.

\section{Fabrication of the probe}

A quartz optical fiber of 1-mm diameter (Showa
Electric Wire \& Cable, SHOWGUIDE SF-S800/1000) was cut to a $30-\mathrm{mm}$ length; both ends were then polished with emery paper (\#1500) and alumina (Fujimi Metapolish No. 4). The polished rod were placed in close contact with a pair of polished $0.5-\mathrm{mm}$ diameter quartz optical fibers (SHOWGUIDE SF-S400/500; 1$\mathrm{m}$ long) within a Teflon tube with a $1-\mathrm{mm}$ bore. The linkage part was then covered with a heat-shrinkable

a

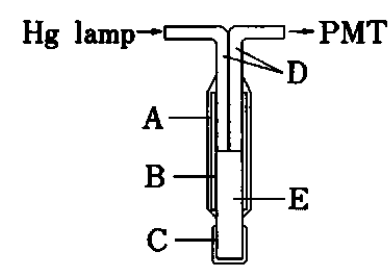

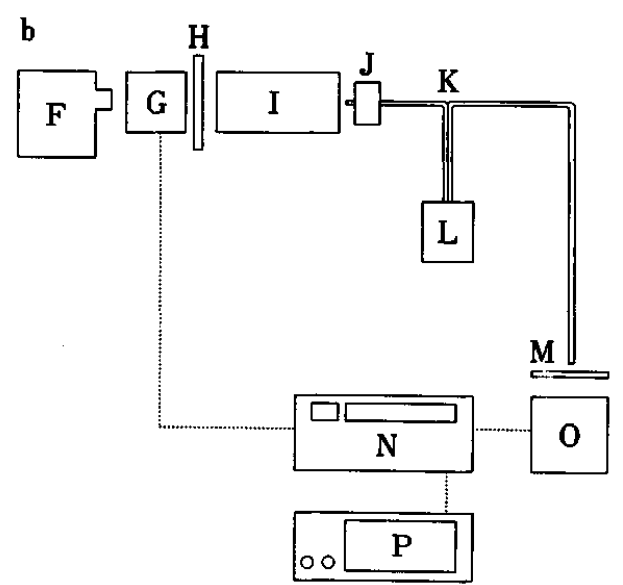

Fig. 1 (a) Construction of the probe and (b) schematic diagram of the instrumentation. A, heat-shrinkable tube; $B$, Teflon tube; C, PVC membrane; D, $0.5 \mathrm{~mm} \phi$ quartz fiber; $\mathrm{E}, 1 \mathrm{~mm} \phi$ quartz fiber; $\mathrm{F}, \mathrm{Hg}$ lamp; G, light chopper; $\mathbf{H}$, interference filter; $\mathbf{I}$, monochromator; $\mathbf{J}$, fiber positioner; $\mathbf{K}$, bifurcated quartz fiber; $\mathbf{L}$, sample cell; $\mathbf{M}$, glass filter; N, lock-in amplifier; O, PMT; P, recorder. 
tube (Fig. 1(a)). The PVC membrane was fixed to the distal end of the fiber-optic sensor by dipping it into $0.5 \mathrm{ml}$ of a THF solution containing $75 \mathrm{mg}$ of NPOE, $25 \mathrm{mg}$ of PVC and $4 \mathrm{mg}$ of PCA; it was then dried under an argon atmosphere at $48 \pm 2^{\circ} \mathrm{C}$ for $4 \mathrm{~h}$. The thickness of the membrane was $6 \pm 2 \mu \mathrm{m}$.

\section{Apparatus and procedure}

Fluorescence and absorption spectra were taken with a Shimadzu fluorescence spectrophotometer (Model RF-510A) and a JASCO spectrophotometer (Model UVIDEC-610A), respectively. The water content was calibrated with a Hiranuma Karl Fischer titration apparatus (Model AQ-5).

A schematic diagram regarding measurements of the fluorescence intensity from the probe is given in Fig. 1(b). The excitation light from an Ushio highpressure Hg lamp (Model USH-500D) was modulated with a light chopper (NF CH-353) and passed through both an interference filter (Toshiba KL-43) and a Shimadzu Baush \& Lomb monochromator (grating 1350 grooves $/ \mathrm{mm}$ ). Light of $430 \mathrm{~nm}$ was admitted into one end of the bifurcated fiber-optic sensor and guided to the probe part, immersed in $10 \mathrm{ml}$ of a sample solution. The collected fluorescence was passed through a glass filter (Toshiba Y-47) and guided to a photomultiplier tube (Hamamatsu R105) operated at $-700 \mathrm{~V}$. The output from the photomultiplier was amplified with an NF lock-in amplifier (Model 5600A) and recorded on a TOA recorder (Model FPR-221A).

The sample solution was magnetically stirred in a light-tight batchwise cell at a temperature maintained at $20.0 \pm 0.5^{\circ} \mathrm{C}$. After the background fluorescence intensity from the probe became constant, water was injected with a microsyringe to the sample solution. For repeated runs, the solution containing water was exchanged for pure ethanol in a separate cell.

\section{Results and Discussion}

Figure 2(a) shows that the acetal form of PCA in acidic ethanol easily reverts to its original aldehyde form, which has absorption bands at wavelengths of $350-400 \mathrm{~nm}$, upon the addition of a small amount of water. A concomitant increase in the fluorescence intensity was observed (Fig. 2(b)). As expected, the fluorescence intensity from the PVC membrane probe was increased by the addition of water. Typical response curves are shown in Fig. 3. The response was accelerated by the presence of $\mathrm{HCl}$ : the time required for a $95 \%$ response was $598 \mathrm{~s}$ in the absence of $\mathrm{HCl}$, while it was $114 \mathrm{~s}$ in the presence of $0.114 \mathrm{M} \mathrm{HCl}$. This phenomenon can be accounted for by invoking acid catalysis, generally recognized in the hydrolysis of acetals. When a sample solution containing water was subsequently replaced by pure ethanol containing $0.114 \mathrm{M} \mathrm{HCl}$, the intensity was reduced to the original level. Though the reproducibility of the response on

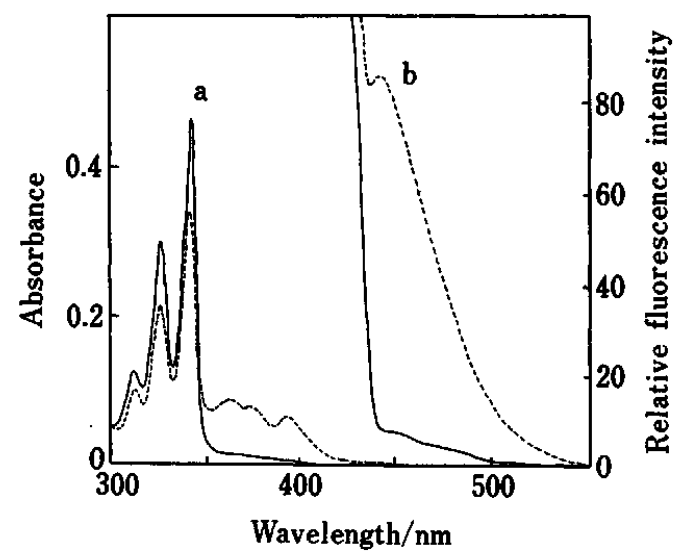

Fig. 2 (a) Absorption and (b) fluorescence spectra of PCA in acidic ethanol $([\mathrm{HCl}]=0.114 \mathrm{M})$ in the absence (full line) or presence (dotted line) of $1 \% \mathrm{H}_{2} \mathrm{O}$. The excitation wavelength for the fluorescence spectra was $430 \mathrm{~nm}$.

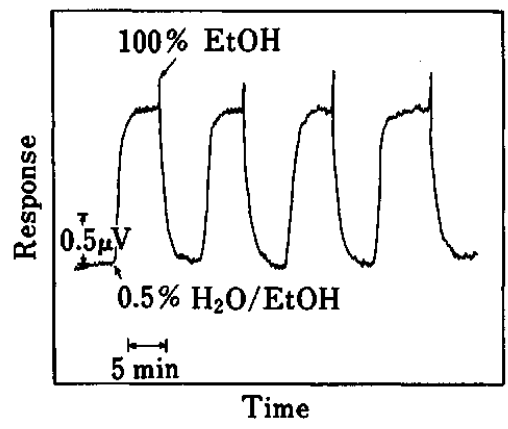

Fig. 3 Typical response of the sensor in acidic ethanol $([\mathrm{HCl}]=0.114 \mathrm{M})$.

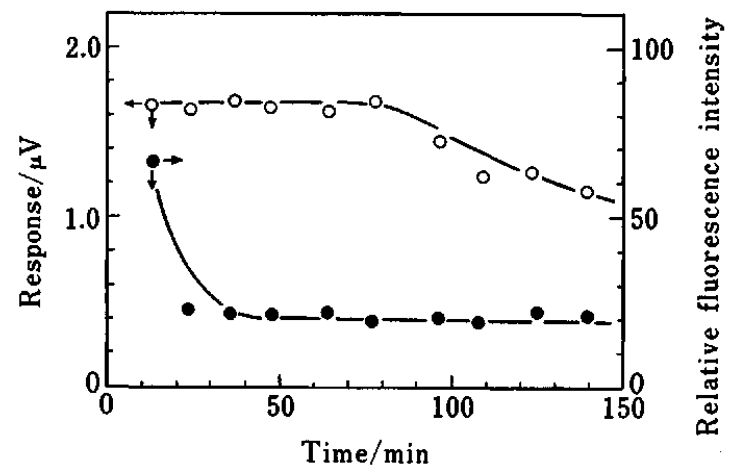

Fig. 4 Time course of the fiber-optic response $(O)$ and the emission from the bulk ethanol (O). On respective run, $0.5 \%$ of $\mathrm{H}_{2} \mathrm{O}$ was injected.

the first five repeated runs was within $3 \%$, the intensity of the response gradually decreased, reaching 75\% of the first response in the 10th run ( $O$ in Fig. 4). A large amount of PCA was eluted from the PVC 


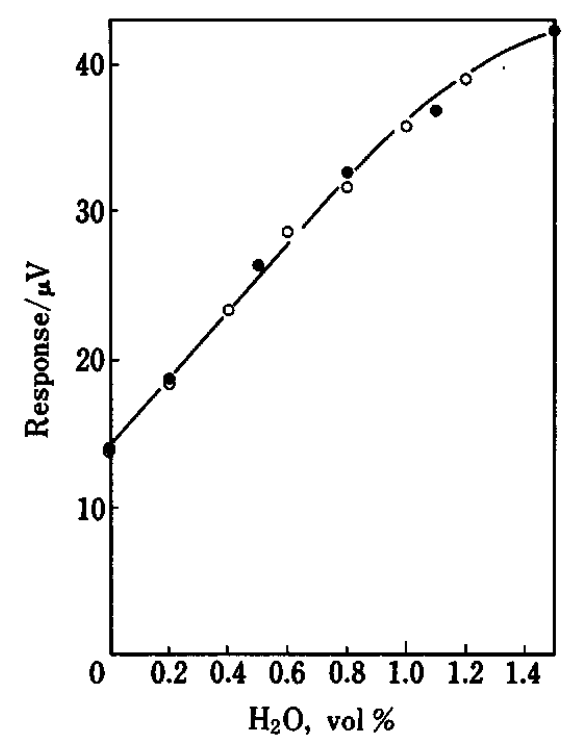

Fig. 5 Relationship between the response and water content in acidic ethanol $([\mathrm{HCl}]=0.114 \mathrm{M}) .(\mathrm{O})$, 1st run; $(\Theta)$, 2nd run. Water was added to ethanol successively and the sample solution was replaced by absolute ethanol containing $0.114 \mathrm{M}$ $\mathrm{HCl}$ after the first run was completed.

membrane to bulk ethanol during the first run; on subsequent runs the PCA concentration in the bulk was kept at a low level ( $\mathrm{in}$ Fig. 4). This result suggests that the response of the probe originates from hydrolysis occurring within the membrane and/or on the membrane surface.
A linear relationship was observed between the fluorescence intensity and water content below about $1 \%$ (Fig. 5). At above $1 \%$ water, the calibration curve became sublinear. This phenomenon is probably due to the limited ability of the plasticized PVC membrane for water uptake. 4,5

The detection limit for the water content was $0.02 \%$ $(S / N=3)$, much lower than that (ca. $0.3 \%$ ) with an optical sensor ${ }^{3}$ developed so far. In addition, the response time (114s) is sufficiently short to apply the present sensor to on-stream analysis. The sensor also has an advantage in that the probe part is readily regenerated by dipping it into a THF solution and drying. The sensor however, has a disadvantage in that PCA elutes gradually. Attempts toward performance improvements are now in progress.

\section{References}

1. T. N. Gvozdovich, G. S. Grinberg, L. V. Zuyeva and Ya. I. Yashin, Petroleum Chem., 12, 120 (1972).

2. J. M. Hogan, R. A. Engel and H. F. Stevenson, Anal. Chem., 42, 249 (1970).

3. K. Matsubara, S. Kawata and S. Minami, Appl. Spectrosc., 42, 1375 (1988).

4. R. D. Armstrong and G. Horvai, Electrochim. Acta, 35, 1 (1990).

5. A. P. Thoma, A. Viviani-Nauer, S. Arvanitis, W. E. Morf and W. Simon, Anal. Chem., 49, 1567 (1977).

(Received September 12, 1990) (Accepted October 17, 1990) 